\title{
Central hypothyroidism or subclinical hyperthyroidism: can they be confused with each other?
}

Mauro Boronat ${ }^{1,2}$

1Section of Endocrinology and Nutrition, Complejo Hospitalario Universitario Insular Materno-Infantil, Las Palmas de Gran Canaria, Spain and ${ }^{2}$ Research Institute in Biomedical and Health Sciences, University of Las Palmas de Gran

Canaria, Las Palmas de Gran Canaria, Spain
Correspondence

should be addressed

to M Boronat

Email

mborcor@gmail.com

\section{Summary}

Isolated, adult-onset central hypothyroidism is very rare, and its diagnosis can be challenging. A 42-year-old patient was referred for evaluation of a $2.8 \mathrm{~cm}$ thyroid nodule. She referred symptoms that could be attributed to hypothyroidism and thyroid tests showed low TSH and normal-low levels of free T4. However, evaluation of the remaining pituitary hormones and pituitary MRI were normal, yet a radionuclide scanning revealed that the thyroid nodule was 'hot' and the tracer uptake in the remaining thyroid tissue was suppressed. Interpretation of these studies led to a misdiagnosis of subclinical hyperthyroidism and the patient was treated with radioiodine. Soon after treatment, she developed a frank hypothyroidism without appropriate elevation of TSH and the diagnosis of central hypothyroidism was made a posteriori. Long term follow-up revealed a progressive pituitary failure, with subsequent deficiency of ACTH and GH. This case should alert to the possibility of overlooking central hypothyroidism in patients simultaneously bearing primary thyroid diseases able to cause subclinical hyperthyroidism.

\section{Learning points:}

- Although rarely, acquired central hypothyroidism can occur in the absence of other pituitary hormone deficiencies.

- In these cases, diagnosis is challenging, as symptoms are unspecific and usually mild, and laboratory findings are variable, including low, normal or even slightly elevated TSH levels, along with low or low-normal concentrations of free T4.

- In cases with low TSH levels, the coexistence of otherwise common disorders able to cause primary thyroid hyperfunction, such as autonomous nodular disease, may lead to a misdiagnosis of subclinical hyperthyroidism.

\section{Background}

Acquired central hypothyroidism usually occurs along with multiple hormone pituitary deficiencies in patients with lesions of the hypothalamic-pituitary region $(1,2)$. However, exceptionally can present in isolation or being the first manifestation of a progressive hypopituitarism (3). In these cases the diagnosis can be particularly challenging, because the clinical manifestations are nonspecific and often milder than in primary hypothyroidism (1). In addition, in most cases, TSH levels are usually normal or even mildly elevated and free T4 levels can be only slightly decreased or even in the lower part of the normal range $(1,2)$. Less often, TSH values can fall below the lower limit of the normal range. Although in these cases the diagnosis should be easier, it could be complicated by confusion with primary subclinical hyperthyroidism in occasional patients who 
simultaneously bear some primary thyroid disorders susceptible to cause autonomous thyroid hyperfunction and suppression of TSH secretion.

\section{Case presentation}

A 42-year-old woman was referred to our Endocrinology Department for evaluation of a thyroid nodule in July 2005. The patient was under study by the Internal Medicine Service because of a several years history of asthenia, feeling of generalized swelling, joints pain, depressive mood and oligomenorrhea. She had undergone standard laboratory tests, which showed normal results, including a TSH measurement $(1.6 \mathrm{mU} / \mathrm{L})$, as well as an abdominal CT scan and, more recently, a cervical ultrasound. In the latter, a well-defined, hypoechoic nodule, measuring $1.8 \times 1.6 \times 2.8 \mathrm{~cm}$, was identified in the left thyroid lobe. Previous personal history only included psoriasis and cervical spondylosis. The patient was not taking drugs interfering with thyroid function.

At the first assessment, the patient weighed $78.3 \mathrm{~kg}$ and measured $162 \mathrm{~cm}$. She presented bilateral palpebral edema and a 1.5-2 cm nodule was confirmed by palpation in the left thyroid lobe. The rest of the physical examination was unremarkable.

\section{Investigation}

The initial diagnostic work-up consisted of a fine-needle aspiration biopsy of the thyroid nodule, which yielded a result of colloid goiter, and repeated thyroid tests, which showed a TSH level of $0.18 \mathrm{mU} / \mathrm{L}$, free T4: $1.0 \mathrm{ng} / \mathrm{dL}$ and negative TPO antibodies $(10.4 \mathrm{U} / \mathrm{mL})$. In view of these findings, central hypothyroidism was suspected, and the study was completed with a pituitary MRI, which did not reveal abnormal findings, and a biochemical assessment of pituitary function, whose results were within the laboratory reference range (Table 1). Subsequently, a ${ }^{99 \mathrm{~m} T c}$ thyroid scintigraphy showed a hot nodule corresponding to the left nodule detected on physical exam and ultrasonography, along with a suppressed activity in the contralateral lobe (Fig. 1). With all this, and despite the fact that the clinical picture was not concordant, it was considered that a diagnosis of subclinical hyperthyroidism secondary to an autonomous thyroid nodule was much more likely than an isolated central hypothyroidism.

\section{Treatment}

Radioiodine treatment was proposed to the patient, but she declined it and remained in follow-up for 2 years. During that time, her free T4 levels were in the lower half of the normal range or even occasionally below the lower limit of normality (between 0.53 and $0.62 \mathrm{ng} / \mathrm{dL}$ ) and TSH progressively decreased, to a minimum of $0.041 \mathrm{mU} / \mathrm{L}$ in June 2007. At that time, she agreed to receive treatment with radioiodine and was given $15 \mathrm{mCi}$ of ${ }^{131} \mathrm{I}$.

\section{Outcome and follow-up}

Two months after treatment, she reported feeling much worse, with extreme tiredness, drowsiness, memory loss and swelling of the face, hands and feet. Physical examination was suggestive of myxedema. A new assessment of thyroid function showed a TSH level of $2.74 \mathrm{mU} / \mathrm{L}$, free T4 $0.40 \mathrm{ng} / \mathrm{dL}$ and total T3 $97.72 \mathrm{ng} / \mathrm{dL}$. Repeated pituitary hormone tests showed normal results, except for low levels of IGF-1 (Table 1). A TRH stimulation test was also performed and revealed a normal TSH response, from baseline levels of $2.17 \mathrm{mU} / \mathrm{L}$ to a peak of $18.26 \mathrm{mU} / \mathrm{L}$ at $30 \mathrm{~min}$ post-stimulation.

Table 1 Most relevant basal hormonal determinations throughout the patient's follow-up.

\begin{tabular}{|c|c|c|c|c|c|c|c|c|c|}
\hline & Mar 2003 & Aug 2005 & Mar 2006 & Nov 2006 & Jun 2007 & Sep 2007 & May 2008 & Jun 2018 & Normal range \\
\hline TSH (mU/L) & 1.6 & 0.18 & 0.09 & 0.055 & 0.041 & 2.74 & 0.02 & 0.01 & $0.34-5.6$ \\
\hline Free T4 (ng/dL) & & 1.00 & 0.60 & 0.53 & 0.62 & 0.40 & 1.48 & 1.19 & $0.6-1.6$ \\
\hline Total T3 (ng/dL) & & & & & 149.07 & 97.72 & & & $80-200$ \\
\hline $\begin{array}{l}\text { Serum cortisol } \\
(\mu g / d L)\end{array}$ & & 17.7 & & & & 9.73 & & 1.56 & $6.7-2.6$ \\
\hline ACTH (pg/mL) & & & & & & & & 1.9 & $10.0-65$ \\
\hline FSH (mU/mL) & & 16.4 & & & & 2.51 & & 44.28 & * \\
\hline LH (mU/mL) & & 3.0 & & & & 2.27 & & 21.19 & * \\
\hline Estradiol (pg/mL) & & 49.7 & & & & 161 & & & * \\
\hline Prolactin (ng/mL) & & 8.9 & & & & 9.88 & & & $3.3-26.7$ \\
\hline IGF-1 (ng/mL) & & 105 & & & & 65 & & 106.3 & $73-287$ \\
\hline
\end{tabular}




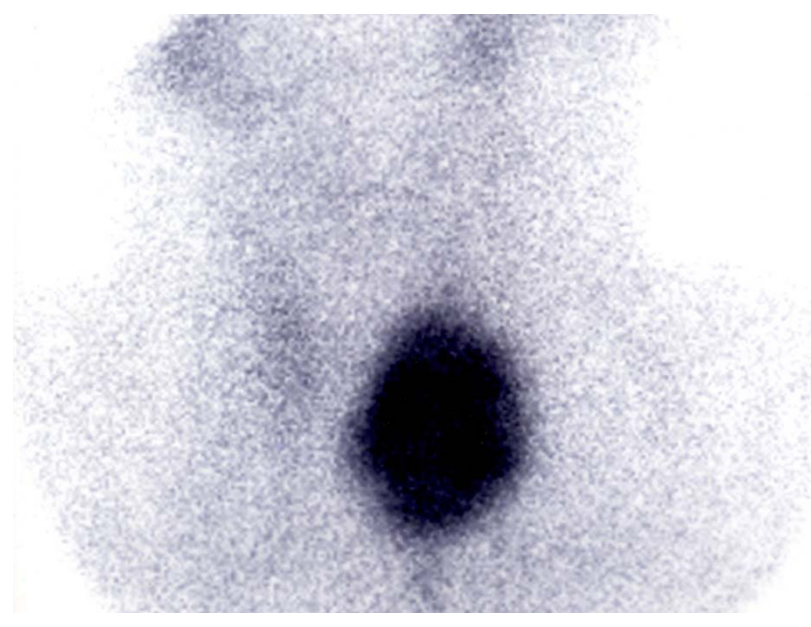

Figure 1

99mTc scan showing a hot thyroid nodule in the left lobe and suppression of radiotracer uptake in the rest of the gland.

A final diagnosis of isolated idiopathic central hypothyroidism combined with an autonomous thyroid nodule was then established. Levothyroxine treatment was prescribed (100 $\mu \mathrm{g}$ per day). The patient experienced a great improvement in her general condition. In May 2008, coinciding with new laboratory tests in which her TSH level was $0.02 \mathrm{mU} / \mathrm{L}$ and her free T4 $1.48 \mathrm{ng} / \mathrm{dL}$, she reported that the symptoms she had been suffering for years had disappeared.

The patient was followed-up annually thereafter. She had menopause in 2014, at age 51. In a scheduled review in June 2018, she reported being very tired and complained of symptoms suggestive of hypoglycemia after longer fasting periods. Biochemical assessment (Table 1) showed normal fasting glucose $(82 \mathrm{mg} / \mathrm{dL})$ and very low levels of ACTH and morning cortisol (measured with an immunoenzimatic assay; Unicel Dxi 800, Beckman Coulter Inc., Brea, CA), while FSH and LH were elevated within physiological postmenopausal ranges. A $250 \mu \mathrm{g}$ ACTH stimulation test showed an apparently normal cortisol response (post-ACTH cortisol concentration of $20.7 \mu \mathrm{g} / \mathrm{dL}$ ). The patient underwent an insulin-induced hypoglycemia test, which revealed blunted responses of both cortisol and GH (post-stimulation peaks of $16.1 \mu \mathrm{g} / \mathrm{dL}$ and $4.9 \mathrm{ng} / \mathrm{mL}$, respectively). A new MRI again did not find structural anomalies of the pituitary gland (Fig. 2) and pituitary antibodies, assayed with a commercial kit test, were negative. The patient began on hydrocortisone (20 mg per day). GH deficiency was confirmed with a glucagon stimulation test (GH peak after stimulation 0.14 $\mathrm{ng} / \mathrm{mL}$ ) and she subsequently was prescribed substitutive GH replacement therapy. A marked improvement was
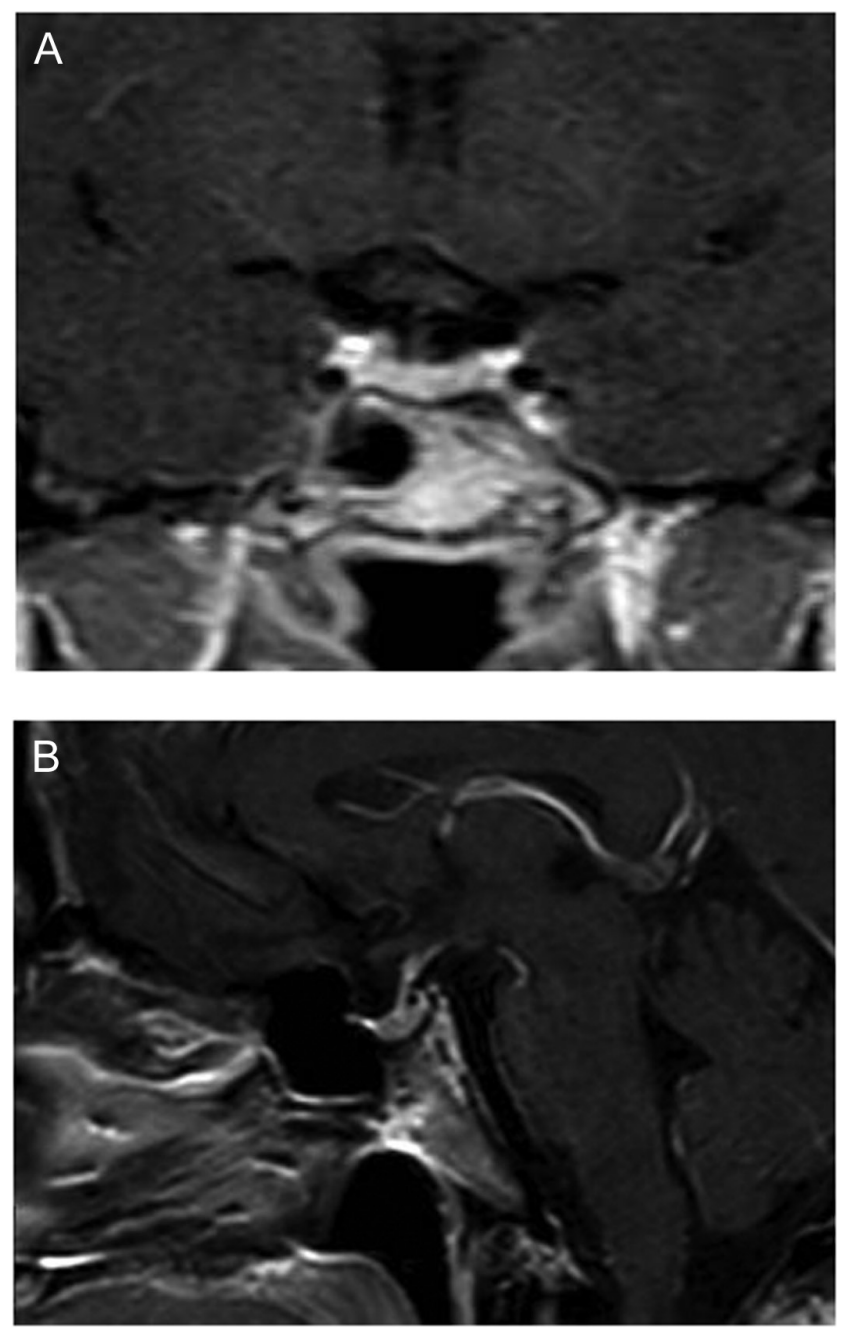

\section{Figure 2}

( $A$ and $B$ ) Contrast enhanced coronal and sagittal sections of the pituitary MRI.

observed with both hydrocortisone and GH replacement therapies. Complete results of hormone dynamic tests are provided as Supplementary material (see section on supplementary materials given at the end of this article).

\section{Discussion}

Central hypothyroidism is an uncommon endocrine disorder in which the diagnosis is often challenging due to the low reliability of clinical and biochemical investigations $(1,2)$.

Sometimes the diagnosis may be delayed or even missed because the general use of TSH-based screening strategies for the diagnosis of hypothyroidism. For example, by using the combined measurement of TSH and T4, one study detected 15 cases of central hypothyroidism (all with normal TSH) over 12 months, testing 56000 
blood samples from a population of 471000 people (4). In other cases, the diagnosis may be complicated by the secretion of bioinactive forms of TSH, which can result in slightly higher-than-normal levels of TSH (5) and lead to a misdiagnosis of subclinical primary hypothyroidism (6).

However, the diagnostic confusion between central hypothyroidism and subclinical hyperthyroidism, as in the present case, is exceptional, and at first sight seems to be counterintuitive. Two factors contributed decisively to this mistake. First, while central acquired hypothyroidism usually occurs in the context of hypopituitarism, in this case it initially developed in the absence of other pituitary hormone deficiencies. According to a literature review, only nine cases of adult-onset isolated central hypothyroidism were published between 1950 and 2015, only five of them outside of Japan (3). Therefore, although the patient's clinical picture was compatible with hypothyroidism and the low TSH values could have suggested a diagnosis of central hypothyroidism, otherwise preserved pituitary function and absence of abnormal findings in the MRI study led to the dismissal of this possibility. Second, the coexistence of an autonomous thyroid nodule, confirmed by the scintigraphic scan, was considered, by itself, as a plausible explanation for the biochemical findings. Most likely, the autonomous thyroid nodule maintained some production of thyroid hormones despite the insufficient TSH stimulation and prevented the patient from developing more profound symptoms of hypothyroidism until she was treated with radioiodine.

The coexistence of a central hypothyroidism and an autonomous thyroid adenoma has been observed previously, at least in one reported case (7), but the diagnosis of hypopituitarism was made, because the patient had a prolactin-secreting pituitary macroadenoma. Having reviewed the literature, to my knowledge there is only two precedents that could bear some resemblance to this case, in that a primary thyroid disease able to cause subclinical hyperthyroidism hindered the detection of a central hypothyroidism. Barbesino et al. (8) reported the case of a woman with undetectable TSH levels, low free T4 and normal total T3, who was diagnosed of painless thyroiditis, on the basis of a history of atrial fibrillation, weakly positive antithyroglobulin antibodies and low radioiodine uptake. However, she had long-lasting symptoms of hypopituitarism and several months later was diagnosed of central hypothyroidism and partial secondary adrenal insufficiency, caused by autoimmune hypophysitis. More recently, Glyn et al. (9) described the case of a patient with Graves' disease treated with subtotal thyroidectomy, who, after the surgery, developed a progressive hypothyroidism that went unnoticed because her TSH levels were normal or low. She was diagnosed with isolated central hypothyroidism with severe complications 20 years later.

Undoubtedly, the main learning message of these cases and the one here reported is that the importance of the anamnesis and clinical findings should never be neglected in favor of apparently incongruent complementary studies. Although obvious, this consideration results particularly easy to overlook in the case of central hypothyroidism, given that the symptoms and the laboratory investigations can be unrevealing. Additionally, this case highlights the fact that isolated central hypothyroidism can be rarely acquired in adulthood, even when it is the only initial manifestation of a slowly progressive multiple pituitary failure. There was no previous history of traumatic brain injury, brain irradiation or intake of drugs able to block TSH secretion. So, even though pituitary antibodies were negative and imaging studies were not characteristic, the most plausible cause for this exceptional presentation of hypopituitarism is lymphocytic hypophysitis, which uses to show a particular propensity to injure TSH-producing cells. Other cases of isolated central hypothyroidism secondary to autoimmune hypophysitis have been described, and, in fact, one study reported a tendency for the rest of the pituitary function to deteriorate over time in some of patients (10).

Supplementary materials

This is linked to the online version of the paper at https://doi.org/10.1530/ EDM-20-0059.

\section{Declaration of interest}

The author declares that there is no conflict of interest that could be perceived as prejudicing the impartiality of the research reported.

\section{Funding}

This research did not receive any specific grant from any funding agency in the public, commercial or not-for-profit sector.

\section{Patient consent}

Written informed consent was obtained from the patient for publication of the submitted article and accompanying images. 
Author contribution statement

Mauro Boronat was the treating physician of the patient and wrote the manuscript.

\section{References}

1 Beck-Peccoz P, Rodari G, Giavoli C \& Lania A. Central hypothyroidism - a neglected thyroid disorder. Nature Reviews: Endocrinology 201713 588-598. (https://doi.org/10.1038/ nrendo.2017.47)

2 Persani L, Cangiano B \& Bonomi M. The diagnosis and management of central hypothyroidism in 2018. Endocrine Connections 20198 R44-R54. (https://doi.org/10.1530/EC-18-0515)

3 Tang C \& Yu R. Adult-onset idiopathic isolatedcentral hypothyroidism: a case report and literature review. Endocrinologia y Nutricion 201562 49-50. (https://doi.org/10.1016/j. endonu.2014.10.001)

4 Wardle CA, Fraser WD \& Squire CR. Pitfalls in the use of thyrotropin concentration as a first-line thyroid-function test. Lancet $2001 \mathbf{3 5 7}$ 1013-1014. (https://doi.org/10.1016/S0140-6736(00)04248-3)

5 Persani L, Ferretti E, Borgato S, Faglia G \& Beck-Peccoz P. Circulating thyrotropin bioactivity in sporadic central hypothyroidism. Journal of Clinical Endocrinology and Metabolism 200085 3631-3635. (https:// doi.org/10.1210/jcem.85.10.6895)

6 Anyiam O, Cheung B \& Al-Sabbagh S. Subclinical hypothyroidism or central hypothyroidism. The danger of thyroid function misinterpretation. Clinical Case Reports 20186 1953-1957. (https:// doi.org/10.1002/ccr3.1694)

7 Foppiani L, Ruelle A, Cavazzani P \& del Monte P. Hyperthyroidism unmasked several years after the medical and radiosurgical treatment of an invasive macroprolactinoma inducing hypopituitarism: a case report. Cases Journal 20092 6449. (https://doi.org/10.4076/17571626-2-6449)

8 Barbesino G, Sluss PM \& Caturegli P. Central hypothyroidism in a patient with pituitary autoimmunity: evidence for TSHindependent thyroid hormone synthesis. Journal of Clinical Endocrinology and Metabolism 201297 345-350. (https://doi. org/10.1210/jc.2011-1591)

9 Glyn T, Harris B \& Allen K. Lessons learnt from a case of missed central hypothyroidism. Endocrinology, Diabetes and Metabolism Case Reports 20172017 17-0112. (https://doi.org/10.1530/EDM-17-0112)

10 Hashimoto K, Yamakita N, Ikeda T, Matsuhisa T, Kuwayama A, Sano T, Hashimoto K \& Yasuda K. Longitudinal study of patients with idiopathic isolated TSH deficiency: possible progression of pituitary dysfunction in lymphocytic adenohypophysitis. Endocrine Journal 200653 593-601. (https://doi.org/10.1507/endocrj.k06-055)

Received in final form 29 May 2020

Accepted 15 June 2020 